\title{
PERFIL EPIDEMIOLÓGICO E SEGUIMENTO APÓS ALTA DE PACIENTES INTERNADOS EM UNIDADE DE TERAPIA INTENSIVA
}

\author{
Jonatas Mendes de Albuquerque ${ }^{1}$, Renata Flavia Abreu da Silva ${ }^{2}$, Ruth Francisca Freitas de Souza ${ }^{3}$
}

\begin{abstract}
RESUMO: Descrever o perfil epidemiológico e o seguimento após alta de pacientes internados em uma unidade de terapia intensiva. Pesquisa descritiva, seccional e de abordagem quantitativa, por meio de análise documental dos prontuários eletrônicos de pacientes adultos, internados na unidade de terapia intensiva em um hospital de atenção terciária na cidade do Rio de Janeiro, no período de novembro de 2014 a novembro de 2015. Na amostra $(n=573) 73 \%$ apresentaram idade igual ou acima de 60 anos, que foi associada a maior risco de morte. A média do tempo de internação na unidade de terapia intensiva foi de 10,7 dias, a taxa de óbito na unidade de terapia intensiva foi de $26 \%(n=148)$, sendo que $56 \%(n=112)$ foram a óbito por choque séptico. Foram readmitidos na unidade de terapia intensiva $10 \%(n=44)$ dos pacientes que apresentaram maior risco relativo para óbito $(p=0,001)$. As variáveis sexo feminino, idade acima de 60 anos, internação na unidade de terapia intensiva acima de 30 dias e readmissão na unidade são fatores de risco para óbito.
\end{abstract}

DESCRITORES: Unidades de terapia intensiva; Readmissão do paciente; Mortalidade hospitalar; Choque séptico.

\section{EPIDEMIOLOGICAL PROFILE AND MONITORING AFTER DISCHARGE OF PATIENTS HOSPITALIZED AT AN INTENSIVE CARE UNIT}

ABSTRACT: To describe the epidemiological profile and monitoring after discharge of patients hospitalized at an intensive care unit. Descriptive and cross-sectional research with a quantitative approach, using documentary analysis of adult patients' electronic histories, who were hospitalized at a tertiary care service in the city of Rio de Janeiro between November 2014 and November 2015 In the sample $(n=573), 73 \%$ were 60 years of age or older, which was associated with a higher risk of death. The mean length of hospitalization at the intensive care unit was 10.7 days, the death rate at the intensive care unit was $26 \%(n=148)$ and $56 \%(n=112)$ died of septic shock. Ten percent $(n=44)$ of patients with relative death risk $(p=0.001)$ were readmitted to the intensive care unit. The variables female sex, age over 60 years, hospitalized at the intensive care unit for more than 30 days and readmission to the unit are risk factors for death.

DESCRIPTORS: Intensive care units; Patient readmission; Hospital mortality; Shock, septic.

\section{PERFIL EPIDEMIOLÓGICO Y SEGUIMIENTO TRAS ALTA DE PACIENTES INTERNADOS EN UNIDAD DE TERAPIA INTENSIVA}

RESUMEN: Describir el perfil epidemiológico y el seguimiento tras alta de pacientes internados en una unidad de terapia intensiva. Investigación descriptiva, seccional y de aproximación cuantitativa, mediante análisis documental de los archivos electrónicos de pacientes adultos, internados en la unidad de terapia intensiva en un hospital de atención terciaria en la ciudad de Rio de Janeiro, entre noviembre del 2014 y noviembre del 2015. En la muestra ( $n=573), 73 \%$ tuvo edad igual o superior a 60 años, que fue asociada a mayor riesgo de muerte. El promedio del tiempo de hospitalización en la unidad de terapia intensiva fue 10.7 días, la tasa de óbito en la unidad de cuidados intensivos fue del $26 \%(n=148)$, siendo que $56 \%(n=112)$ fallecieron por choque séptico. Fue readmitido en la unidad de terapia intensiva $10 \%(n=44)$ de los pacientes que presentaron mayor riesgo relativo para óbito $(p=0,001)$. Las variables sexo femenino, edad superior a 60 años, hospitalización en la unidad de cuidados intensivos superior a 30 días y readmisión en la unidad son factores de riesgo para óbito.

DESCRIPTORES: Unidades de cuidados intensivos; Readmisión del paciente; Mortalidad hospitalaria; Choque séptico.

${ }^{1}$ Enfermeiro. Residente de Clínica médica da Universidade Federal do Estado do Rio de Janeiro. Rio de Janeiro, RJ, Brasil.

${ }^{2}$ Enfermeira. Doutora em Ciências. Docente de Enfermagem da Universidade Federal do Estado do Rio de Janeiro. Rio de Janeiro, RJ, Brasil.

${ }^{3}$ Enfermeira. Mestre em Enfermagem. Enfermeira na Unidade de Terapia Intensiva do Hospital Naval Marcílio Dias-Marinha do Brasil. Rio de Janeiro, RJ, Brasil. 


\section{- INTRODUÇÃO}

As Unidades de Terapia Intensiva (UTI) constituem espaços especializados em hospitais destinados ao tratamento de pacientes cuja sobrevivência se encontra ameaçada por doenças ou condições clínicas que causam instabilidade ou disfunção de um ou mais sistemas fisiológicos. Para prestar assistência adequada a esses pacientes, são necessários, além de pessoal qualificado, recursos tecnológicos de monitorização, suporte de funções vitais, uso de dispositivos invasivos, e conhecimento da necessidade dos pacientes ${ }^{(1)}$.

Entre as maneiras disponíveis para conhecer a necessidade dos pacientes, destaca-se a realização de pesquisas, como de perfil epidemiológico, a qual engloba um conjunto de ações que proporcionam o conhecimento, a detecção ou prevenção de qualquer mudança nos fatores determinantes e condicionantes de saúde individual ou coletiva, com a finalidade de recomendar e adotar as medidas de prevenção e controle das doenças ou $\operatorname{agravos}^{(2)}$.

Por isso, o perfil epidemiológico é um importante instrumento para o planejamento, a organização e a operacionalização dos serviços de saúde, como também para a normatização de atividades técnicas correlatas $^{(2)}$. A pesquisa também se mostra relevante a partir do momento que ocorrem mudanças na dinâmica do perfil epidemiológico das doenças e avanços do conhecimento científico, fato que torna necessária a atualização constante do conhecimento epidemiológico da população a ser pesquisada ${ }^{(2)}$.

Além disso, o meio pelo qual pode-se aprofundar o conhecimento do perfil epidemiológico do paciente consiste na avaliação do seu seguimento, através do acompanhamento do paciente durante todo o período de internação hospitalar. A unidade de destino e a evolução da gravidade do paciente na UTI são parâmetros também importantes para facilitar a gestão dos serviços no sentido de oferecer indícios para a continuidade do tratamento do paciente ${ }^{(3)}$.

Conhecer a população assistida na UTI e a sua evolução clínica é de suma importância, pois possibilita detectar mudanças e formular objetivos e metas para o setor, a fim de gerar melhorias na assistência prestada e, consequentemente, nestes indicadores.

Sendo assim, realizaram-se os seguintes questionamentos: qual é o perfil epidemiológico de pacientes na UTI em um hospital de atenção terciária? E o seu seguimento, após a alta da UTI? Para responder a essas questões, a pesquisa teve como objetivo: descrever o perfil epidemiológico e o seguimento após alta de pacientes internados em uma unidade de terapia intensiva de um hospital de atenção terciária.

\section{MÉTODO}

Trata-se de uma pesquisa descritiva do tipo seccional, com análise retrospectiva dos prontuários, e de abordagem quantitativa.

Foi desenvolvida na UTI de um hospital de atenção terciária, no estado do Rio de Janeiro, classificada como clínica geral e cirúrgica adulto, composta por 16 leitos; no período de novembro de 2014 a novembro de 2015, por meio de análise documental dos prontuários eletrônicos dos pacientes admitidos durante este período. Foram incluídos na pesquisa todos os pacientes com idade $\geq 18$ anos, que permaneceram no mínimo 24 horas na UTI e que tiveram seu histórico de internação concluído. Em contrapartida, foram excluídos da pesquisa todos os pacientes que não se apresentavam dentro dos critérios de inclusão anteriormente citados, ou que tinham informações ausentes necessárias à pesquisa, em seu prontuário.

O instrumento para coleta de dados apresentou as variáveis de sexo (masculino e feminino) e idade; procedência: emergência, centro cirúrgico, unidade semi-intensiva, enfermarias; causa de admissão: instabilidade hemodinâmica, instabilidade respiratória, hemodiálise, pós-operatório eletivo, ou de urgência/emergência, alteração neurológica ou outras causas; diagnóstico principal; tempo de internação na UTI em dias; desfecho na UTI: alta, transferência para outro setor ou óbito; seguimento em outro setor: transferência para unidade semi-intensiva, transferência para enfermaria ou transferência para outro local; desfecho em outro setor: alta, óbito ou readmissão na UTI; desfecho 
após a readmissão na UTI (alta ou óbito), e caso haja, a causa do óbito.

Os achados foram organizados e apresentados em tabelas, sendo usado o programa Epi Info versão 7, para a organização dos dados e sua análise por meio do teste do Qui-quadrado e da estatística descritiva, considerando-se $p<0,05$ como significância estatística.

A presente pesquisa foi submetida ao Comitê de Ética em Pesquisa das instituições proponente e co-participante para apreciação e autorização da coleta, segundo a Resolução $n^{\circ} 466 / 12^{(4)}$, sendo aprovada pelos números de pareceres 1.411.041/2016 e 1.446.098/2016, respectivamente.

\section{- RESUlTADOS}

Foram considerados 600 prontuários, conforme critérios descritos, contudo 27 foram excluídos por apresentarem tempo de internação menor de 24 horas na UTI, totalizando como amostra final 573 prontuários.

A seguir a Tabela 1 apresenta as características demográficas e clínicas da população estudada $(\mathrm{n}=$ 573). Além dos dados categorizados, em relação à idade, a média e a mediana foi de 66,5 anos $\pm 19,4$ e 71 anos respectivamente, e a média de dias de internação foi de 10,7 dias $\pm 18,8$ e a mediana de quatro dias.

Tabela 1 - Características demográficas e clínicas. Rio de Janeiro, RJ, Brasil, 2016 (continua)

\begin{tabular}{lc} 
Características & n (\%) \\
\hline Idade & $70(12)$ \\
\hline$\geq 18$ e $\leq 39$ & $83(15)$ \\
\hline$\geq 40$ e $\leq 59$ & $420(73)$ \\
\hline Sexo & \\
\hline Masculino & $305(53)$ \\
\hline Feminino & $268(47)$ \\
\hline Procedência & \\
\hline Emergência & $254(44)$ \\
\hline Centro Cirúrgico & $241(42)$ \\
\hline Enfermarias & $64(11)$ \\
\hline Unidade Semi-Intensiva & $14(03)$ \\
\hline Causas de admissão & \\
\hline Pós-operatório eletivo & $155(27)$ \\
\hline Insuficiência respiratória & $151(26)$ \\
\hline Pós-operatório urgência/emergência & $88(16)$ \\
\hline Alteração neurológica & $76(13)$ \\
\hline Instabilidade hemodinâmica & $65(11)$ \\
\hline Hemodiálise & $16(03)$ \\
\hline Outra causa & $22(04)$ \\
\hline Diagnóstico principal & \\
\hline Doenças neurológicas & $108(19)$ \\
\hline Doenças do aparelho respiratório & $100(17)$ \\
\hline Neoplasias & $81(14)$ \\
\hline Doenças do aparelho cardiovascular & $57(10)$ \\
\hline Doenças osteoarticulares & \\
\hline
\end{tabular}


Cogitare Enferm. (22)3: e50609, 2017

\begin{tabular}{lc}
\hline Doenças do aparelho gastrointestinal & $53(09)$ \\
\hline Doenças do aparelho geniturinário & $33(06)$ \\
\hline Doenças do sistema endócrino & $20(04)$ \\
\hline Doenças do sistema hepático & $13(02)$ \\
\hline Doenças hematológicas & $10(02)$ \\
\hline Outros & $37(06)$ \\
\hline Tempo de internação na UTI & $198(35)$ \\
\hline$\geq 1$ e $\leq 2$ dias & $187(33)$ \\
\hline$\geq 3$ e $\leq 7$ dias & $64(11)$ \\
\hline$\geq 8$ e $\leq 12$ dias & $35(06)$ \\
\hline$\geq 13$ e $\leq 17$ dias & $19(03)$ \\
\hline$\geq 18$ e $\leq 23$ dias & $11(02)$ \\
\hline$\geq 24$ e $\leq 29$ dias & $59(10)$ \\
\hline$\geq 30$ dias & $148(26)$ \\
\hline Desfecho ao final da $1^{a}$ internação & $425(74)$ \\
\hline Óbito & $112(56)$ \\
\hline Transferidos para outros setores & $40(20)$ \\
\hline Causa do óbito & $12(24)$ \\
\hline Choque séptico & \\
\hline Choque refratário/parada cardiorrespiratória \\
\hline Insuficiência Respiratória
\end{tabular}

Em relação à variável diagnóstico principal, vale ressaltar que a instituição onde foi realizada a pesquisa dispõe de uma unidade de terapia intensiva coronariana, o que justifica um menor número de admissão de pacientes com o diagnóstico principal de doenças cardiovasculares na UTI em apreço.

A Tabela 2 mostra os desfechos relacionados à variável primeira internação, em que se observa como significativo o sexo feminino e idade maior que 60 anos como fatores que levaram a um maior número de óbitos em indivíduos com estas características.

Tabela 2 - Associação no desfecho da 1ª internação na UTI conforme sexo e idade. Rio de Janeiro, RJ, Brasil, 2016

\begin{tabular}{|c|c|c|c|c|c|}
\hline Características & Óbito n (\%) & Transferência & Total & Valor de $p$ & Risco Relativo \\
\hline Feminino & $80(30)$ & $188(70)$ & $268(100)$ & $0.02 *$ & $\mathrm{RR}=1.339 *$ \\
\hline Masculino & $68(22)$ & $237(78)$ & $305(100)$ & & \\
\hline$\geq 60$ anos de idade & $133(32)$ & $287(68)$ & $420(100)$ & $<0.001^{*}$ & $\mathrm{RR}=3.23^{*}$ \\
\hline$\geq 18$ e $\leq 59$ anos de idade & $15(10)$ & $138(90)$ & $153(100)$ & & \\
\hline
\end{tabular}

*Qui-quadrado

A Tabela 3 destaca a associação entre o tempo de internação na UTI e o desfecho na mesma, aonde os pacientes que permaneceram internados acima de 30 dias na unidade em apreço tiveram maior risco relativo de ir a óbito. 
Tabela 3 - Associação no desfecho da $1^{\mathrm{a}}$ internação na UTI, conforme o período de internação na unidade. Rio de Janeiro, RJ, Brasil, 2016

\begin{tabular}{|c|c|c|c|c|c|}
\hline Tempo de Internação & Óbito n (\%) & Transferência & Total & Valor de $p$ & Risco Relativo \\
\hline$\geq 30$ dias & $23(39)$ & $36(61)$ & $59(100)$ & $0.009^{*}$ & $\mathrm{RR}=1.60^{*}$ \\
\hline$\leq 29$ dias & $125(24)$ & 389 (76) & $514(100)$ & & \\
\hline
\end{tabular}

${ }^{*}$ Qui-quadrado

A Tabela 4 evidencia um número significativamente maior de óbitos em outras unidades, após a alta da UTI, entre os pacientes que tiveram tempo de internação acima de 30 dias na UTI durante a primeira internação. Além disso, os mesmos pacientes tiveram maior risco relativo de readmissão na UTI.

Tabela 4 - Associação do desfecho após a alta da UTI. Rio de Janeiro, RJ, Brasil, 2016

\begin{tabular}{|c|c|c|c|c|c|}
\hline $\begin{array}{l}\text { Tempo de Internação } \\
\text { na UTI, durante a } 1^{a} \\
\text { internação }\end{array}$ & $\begin{array}{c}\text { Após a alta da UTI, } \\
\text { Óbito em outras } \\
\text { unidades n (\%) } \\
\end{array}$ & $\begin{array}{c}\text { Após a alta da UTI, } \\
\text { Alta de outras } \\
\text { unidades } \\
\end{array}$ & Total & Valor de $p$ & $\begin{array}{c}\text { Risco } \\
\text { Relativo }\end{array}$ \\
\hline$\geq 30$ dias & $11(38)$ & $18(62)$ & 29 (100) & $<0.001^{*}$ & $\mathrm{RR}=6.069^{*}$ \\
\hline$\leq 29$ dias & $22(06)$ & $330(94)$ & $352(100)$ & & \\
\hline $\begin{array}{l}\text { Tempo de Internação } \\
\text { na UTI, durante a } 1^{a} \\
\text { internação }\end{array}$ & Readmissão na UTI & $\begin{array}{c}\text { Após a alta da UTI, } \\
\text { Alta de outras } \\
\text { unidades } \\
\end{array}$ & Total & Valor de $p$ & $\begin{array}{c}\text { Risco } \\
\text { Relativo }\end{array}$ \\
\hline$\geq 30$ dias & $07(28 \%)$ & $18(72)$ & $25(100)$ & $0.008^{*}$ & $\mathrm{RR}=2.777^{*}$ \\
\hline$\leq 29$ dias & $37(10 \%)$ & $330(90)$ & $367(100)$ & & \\
\hline
\end{tabular}

*Qui-quadrado

Outro fator visto na presente pesquisa foi o maior número de óbitos em pacientes que reinternaram na UTI, que tinham risco ( $R R=1.848)$ maior se comparados aos pacientes que haviam sido internados na primeira vez, comprovado pelos dados na Tabela 5.

Tabela 5 - Comparação do desfecho na $1^{\mathrm{a}}$ internação na UTI com o desfecho na readmissão na UTI. Rio de Janeiro, RJ, Brasil, 2016

\begin{tabular}{|c|c|c|c|c|c|}
\hline Desfechos & Óbito n (\%) & Alta & Total & Valor de p & Risco Relativo \\
\hline Readmissão & $21(48)$ & $23(52)$ & $44(100)$ & $0.001^{*}$ & $\mathrm{RR}=1.848^{*}$ \\
\hline $1^{\mathrm{a}}$ internação & $148(26)$ & $425(74)$ & $573(100)$ & & \\
\hline
\end{tabular}

*Qui-quadrado

\section{- DISCUSSÃO}

Conforme a Tabela 1, em relação às características demográficas, a população total pesquisada (n $=573)$ constitui-se de $53 \%(n=305)$ do sexo masculino e $47 \%(n=268)$ do sexo feminino e $73 \%(n$ = 420) apresentaram mais de 60 anos de idade, sendo que a média e a mediana da idade foram de 66,5 e 71 anos, respectivamente. Esses dados corroboram com pesquisas anteriores, as quais também apresentaram uma maior população de sexo masculino, com uma porcentagem variando de $54 \%$ a $60 \%$, e uma população com idade superior a 60 anos, com a porcentagem variando de $53 \%$ a $58 \%$ (55-8) . 
Quanto à procedência dos pacientes, a maioria teve acesso à UTI a partir do setor de emergência $44 \%(n=254)$, seguido do centro cirúrgico $42 \%(n=241)$. Essa variável está de acordo com algumas pesquisas, aonde o setor de emergência foi o que apresentou a maior taxa de procedência dos pacientes para a UTI, com a porcentagem variando de $26 \%$ a $40 \%{ }^{(6,9-10)}$ mas diverge de outras pesquisas, em que a maioria dos pacientes teve acesso à UTI a partir do centro cirúrgico, com a porcentagem variando de $36 \%$ a $55 \%(3,7-8)$.

Com relação às causas de admissão na UTI, a maior causa foi decorrente de pós-operatório eletivo $27 \%(n=155)$, seguido de insuficiência respiratória $26 \%(n=151)$. Fato que diverge de outra pesquisa em que a maior causa foi insuficiência respiratória (43\% do total de 67 ) seguido de insuficiência hemodinâmica $(29 \% \text { do total de } 67)^{(6)}$.

A categoria mais frequente dentre o diagnóstico principal foi relacionada às doenças neurológicas $19 \%(n=108)$, seguida das doenças do aparelho respiratório $17 \%(n=100)$, e das neoplasias $14 \%(n$ = 81). Esses dados divergiram de outras pesquisas, sendo que as mesmas também não apresentaram concordância em seus resultados referente ao diagnóstico principal; nelas as porcentagens das doenças do aparelho respiratório variaram de $13 \%$ a $31 \%$ e as neoplasias de $10 \%$ a $18 \%{ }^{(6,9)}$.

Em relação ao tempo de internação na UTI, durante a 1a internação, a média foi de 10,7 dias, a mediana de quatro dias e o período predominante foi de 01 a 02 dias $35 \%$ ( $n=198$ ), sendo que $10 \%$ $(\mathrm{n}=59)$ dos pacientes passaram mais de 30 dias na UTI. Na literatura há grandes diferenças no tempo de permanência do paciente na UTI, apresentando uma média de dias de internação variando de 8,9 a 23,2 dias, sendo que as pesquisas convergiram apresentando uma menor porcentagem de pacientes internados com mais de 30 dias na UTI ${ }^{(5,7,9)}$.

Além disso, foi observado que a variável idade $\geq 60$ anos apresentou ser fator de risco $R R=1.78$ $(p=0.03)$, em comparação à variável pacientes $\geq 18$ anos $a \leq 59$ anos de idade, para maior tempo de internação na UTI ( $\geq 30$ dias).

A liberação do paciente da UTI ocorre quando a condição hemodinâmica é estabilizada e não há mais a necessidade de ventilação mecânica invasiva ou de monitorização contínua e cuidados intensivos ${ }^{(8)}$.

Dos pacientes que foram transferidos para outras unidades $(n=425,74 \%$ do total), após a assistência na UTI, 94\% $(n=398)$ foram transferidos para as enfermarias e somente $6 \%(n=27)$ foram para unidade semi-intensiva. Deve-se ressaltar que pesquisas aonde a maior porcentagem da população de pacientes que foram transferidos para as enfermarias em comparação com as unidades semi-intensivas tendem a apresentar um maior tempo de internação na UTI(11). Assim, a presente pesquisa apresentou uma média de internação na UTI de 10,7 dias, em divergência, pesquisas que tiveram maior transferência de pacientes para a unidade semi-intensiva apresentaram médias de 8,1 a 8,9 dias de internação na UTI ${ }^{(3,9)}$.

No Brasil, o número de leitos de unidade semi-intensiva não é conhecido, mas sabe-se que está longe de atender às necessidades da população. Em decorrência disso, quando esses pacientes são internados em enfermarias, não recebem o suporte, os cuidados e o atendimento de pessoal qualificado para assisti-los, o que pode determinar maiores taxas de reinternação em UTI e mortalidade hospitalar $^{(12)}$.

Outro dado relevante é a mortalidade, já que é um frequente indicador de qualidade de assistência em UTI ${ }^{(3)}$. Dos 573 pacientes pesquisados, 148 morreram durante a $1^{\mathrm{a}}$ internação na UTI, totalizando $26 \%$ da amostra. Na literatura essa taxa variou de $11 \%$ a $34,8 \%{ }^{(3,6-7,9-10,12)}$. Esses pacientes apresentaram maior média de 16 dias de internação e maior mediana de 7 dias de internação, quando comparados à população geral.

Ainda em relação ao óbito durante a internação na UTI, pode-se observar na Tabela 3 que os pacientes que passaram $\geq 30$ dias na UTI tiveram um $R R=1.60$ maior $(p=0.009$ ) de ir a óbito quando comparados aos demais. É de consenso, na literatura, que pacientes que permanecem por períodos mais prolongados em UTI são aqueles que apresentam condições mais instáveis ou complicações, decorrentes de sua doença ou procedimentos invasivos ${ }^{(9)}$. Também há pesquisas em países latinoamericanos e europeus que demonstraram a relação entre longas internações e o desenvolvimento de infecções, fator que aumenta a probabilidade de óbito(13-15). 
Nessa pesquisa, de acordo com a Tabela 2, também foi verificado que os pacientes $\geq 60$ anos de idade tiveram risco relativo maior de $R R=3.23(p=<0.001)$ de ir a óbito durante a $1^{a}$ internação na UTI, quando comparados aos demais. Esse dado corroborou com outra pesquisa no qual $63 \%$ dos pacientes $\geq 60$ anos de idade foram a óbito ${ }^{(5)}$. A Tabela 2 demonstrou que os pacientes de sexo feminino também tiveram risco relativo maior de $\mathrm{RR}=1.339(\mathrm{p}=0.02)$ de ir a óbito durante a $1^{\mathrm{a}}$ internação na UTI, quando comparados aos de sexo masculino, fato que diverge de uma pesquisa que não obteve significância estatística entre os sexos, apresentando a porcentagem de óbito do sexo feminino de $16 \%$ e do sexo masculino de $11 \%{ }^{(16)}$.

O óbito também pode ocorrer após a alta da UTI, sendo que dos 425 pacientes transferidos para outros setores, $8 \%(n=33 \%)$ foram a óbito. Com relação a esses pacientes, pode-se observar na Tabela 4 aqueles que passaram $\geq 30$ dias internados na UTI, durante a $1^{a}$ internação, tiveram um RR=6.069 maior ( $p=<0.001$ ) de ir a óbito em outros setores, quando comparados aos demais. Na literatura podese encontrar taxas de óbito após a alta da UTI que variam de $2 \%$ a $4 \%(5,10)$.

Foi verificado que a maior quantidade de óbitos na UTI foi atribuída ao choque séptico 56\% ( $\mathrm{n}=$ 112), seguido de choque refratário/parada cardiorrespiratória $20 \%(n=40)$ e insuficiência respiratória $12 \%$ ( $n=24)$. Esses dados estão de acordo com a literatura, que também apresentou uma maior taxa de óbitos na UTI atribuída ao choque séptico, seguido de choque refratário/parada cardiorrespiratória e insuficiência respiratória ${ }^{(10)}$.

Devemos considerar a possibilidade que a maior parte dos casos classificados como choque refratário e parada cardiorrespiratória podem também ter ocorrido em consequência da sepse ${ }^{(10)}$.

O óbito pode estar relacionado à evolução natural da doença, quando todas as possibilidades terapêuticas já foram esgotadas. Porém, pode também ser decorrente de fatores como a limitação de recursos humanos e de disponibilidade de equipamentos, principalmente em serviços que não possuem unidades de cuidados intermediários ou, ainda, pode indicar alta prematura da UTI(3,8).

A partir disso, considerando os vários fatores limitantes na área da saúde, foi verificado que $10 \%$ do total de 425 pacientes tiveram que ser readmitidos na UTI, sendo que uma revisão de literatura apresentou as taxas de reinternação variando entre 0,89 a $19 \%{ }^{(17)}$. Também foi observado na Tabela 4 que os pacientes que passaram $\geq 30$ dias internados na UTI durante a $1^{\text {a }}$ internação, tiveram um $R R=2.777$ maior $(p=0.008)$ de serem readmitidos na UTI quando comparados aos demais.

Quando a readmissão do paciente ocorre na UTI, novas exposições a procedimentos invasivos ocorrem, por isso, os mesmos são considerados mais graves, apresentam um tempo de internação 2,5 vezes maior, apresentam mais comorbidades crônicas e uma probabilidade de óbito quatro vezes maior comparativamente aos pacientes não readmitidos ${ }^{(18-20)}$.

Foi constatada (Tabela 5) uma elevada taxa de mortalidade de $48 \%$ (do total de 44) após a readmissão de pacientes na UTI, na literatura essa taxa variou entre $27 \%$ e $69 \%^{(20-21)}$. Sendo assim, essa população teve um $R R=1.848(p=0.001)$ maior de ir a óbito comparado com os pacientes durante a $1^{\mathrm{a}}$ internação.

Ressalta-se que a presente pesquisa possui limitações que devem ser consideradas: foi desenvolvida em uma única UTI, todos os pacientes da amostra são de um período de 1 ano, não houve a análise da gravidade clínica dos pacientes, não foi verificado o tempo de internação hospitalar antes da admissão na UTI, o que pode ser um fator para aumento da mortalidade ${ }^{(22)}$. Não foi possível coletar a variável comorbidades de forma satisfatória, pela falta de informações no prontuário, sendo então excluída da pesquisa para que não prejudicasse o resultado da pesquisa.

\section{CONCLUSÃo}

A falta de leitos de unidade semi-intensiva pode ter aumentado a média de dias de permanência na UTI, evidenciando a necessidade de outras pesquisas complementares em duas ou mais instituições que apresentem situações distintas de unidade semi-intensiva, para que essa questão seja melhor elucidada.

As variáveis sexo feminino, idade acima de 60 anos, internação na UTI superior a 30 dias e readmissão 
na unidade foram fator de risco para óbito. A maior causa de óbito por choque séptico, apresentada na pesquisa, está de acordo com a literatura. Porém, não há como afirmar que a causa de óbito foi somente o choque séptico ou se este foi mais um fator que contribuiu para a ocorrência do óbito, por conta do método de coleta utilizado que não permitiu um estudo mais apurado de cada paciente.

Também foi observado que pacientes com idade acima de 60 anos apresentaram fator de risco para necessidade de maior tempo de internação na UTI ( $\geq 30$ dias). Além disso pacientes que passaram mais dias internados na UTI ( $\geq 30$ dias) apresentaram maior porcentagem de óbito em outros setores após a transferência e maior porcentagem de readmissões.

Não havendo a pretensão de esgotar o tema, este trabalho soma conhecimento acerca do perfil epidemiológico dos pacientes em uma UTI e dá suporte para futuras pesquisas na área.

\section{- REFERÊNCIAS}

1. Oliveira FJG, Siqueira JF, Ramos IC, Campos FA, Oriá MOB, Caetano JA. Utilização de cateter venoso central em pacientes internados em uma unidade de terapia intensiva. Rev. Rene. [Internet] 2013;14(5) [acesso em 08 mar 2016]. Disponível: http://dx.doi.org/10.15253/rev\%20rene.v14i5.3614.

2. Ministério da Saúde (BR). Secretaria de Vigilância em Saúde. Departamento de Vigilância Epidemiológica. Guia de vigilância epidemiológica. Brasília: Ministério da Saúde; 2009.

3. de Oliveira VCR, Nogueira LS, Andolhe R, Padilha KG, de Sousa RMC. Clinical evolution of adult, elderly and very elderly patients admitted in Intensive Care Units. Rev Latino-Am. Enfermagem. [Internet] 2011;19(6) [acesso em 08 mar 2016]. Disponível: http://dx.doi.org/10.1590/S0104-11692011000600010.

4. Ministério da Saúde (BR). Conselho Nacional de Saúde. Diretrizes e normas regulamentadoras de pesquisa envolvendo seres humanos. Resolução n. 466, de 12 de dezembro de 2012. Brasília; 2012.

5. de Freitas ERFS. Profile and Severity of the Patients of Intensive Care Units: Prospective Application of the APACHE II. Rev Latino-Am. Enfermagem. [Internet] 2010;18(3) [acesso em 08 mar 2016]. Disponível: http://dx.doi. org/10.1590/S0104-11692010000300004.

6. Vieira MS. Perfil geográfico e clínico de pacientes admitidos na UTI através da Central de Regulação de Internações Hospitalares. Com. Ciências Saúde. [Internet] 2011;22(3) [acesso em 08 mar 2016]. Disponível: http:// bvsms.saude.gov.br/bvs/periodicos/revista_ESCS_v22_n3_a02_Perfil_geografico_clinico.pdf.

7. da Silva LC, Nogueira LS, Settervall CHC, de Sousa RMC, Padilha KG. The use of severity indexes to estimate the risk of death in Intensive Care. Rev. esc. enferm. USP. [Internet] 2012;46(4) [acesso em 15 jul 2016]. Disponível: http://dx.doi.org/10.1590/S0080-62342012000400009.

8. Cardoso LGS, Chiavone PA. The APACHE II measured on patients' discharge from the Intensive Care Unit in the prediction of mortality. Rev. Latino-Am. Enfermagem. [Internet] 2013;21(3) [acesso em 08 mar 2016]. Disponível: http://dx.doi.org/10.1590/S0104-11692013000300022.

9. da Silva MCM, de Sousa RMC, Padilha KG. Fatores associados ao óbito e a readmissão em Unidade de Terapia Intensiva. Rev. Latino-Am. Enfermagem. [Internet] 2011;19(4) [acesso em 08 mar 2015]. Disponível: http://www. scielo.br/pdf/rlae/v19n4/pt_09.

10. Giacomini MG, Lopes MVCA, Gandolfi JV, Lobo SMA. Septic shock: a major cause of hospital death after intensive care unit discharge. Rev Bras Ter Intensiva. [Internet] 2015;27(1) [acesso em 24 jan 2015]. Disponível: http://dx.doi.org/10.5935/0103-507X.20150009.

11. de Sousa CR, Gonçalves LA, Toffoleto MC, Leão K, Padilha KG. Predictors of nursing workland in elderly patients admited to Intensive Care Units. Rev. Latino-Am. Enfermagem. [Internet] 2008;16(2) [acesso em 08 mar 2015]. Disponível: http://dx.doi.org/10.1590/S0104-11692008000200008.

12. Caldeira VMH, Silva Júnior JMS, de Oliveira AMRR, Rezende S, de Araújo LAG, Santana MRO, et al. Criteria for patient ad Mission to in intensive Care unit and related Mortality Rates. Rev. Assoc. Med. Bras. [Internet] 2010;56(5) [acesso em 08 mar 2015]. Disponível: http://dx.doi.org/10.1590/S0104-42302010000500012. 
13. Martins MA. Conceitos gerais e terminologia básica em epidemiologia hospitalar e controle de infecção. In: Martins MA. Manual de infecção hospitalar: epidemiologia, prevenção e controle. Belo Horizonte: Medsi; 2001. p. 16-26.

14. Rosenthal VD, Guzman S, Orellano PW. Nosocomial infections in medical-surgical intensive care units in Argentina: attributable motality and lenght of stay. Am J Infect Control. [Internet] 2003;31(5) [acesso em 08 mar 2015]. Disponível: http://dx.doi.org/10.1067/mic.2003.1.

15. Orsi GB, Scorzolini L, Franchi C, Mondillo V, Rosa G, Venditti M. Hospital-acquired infection surveillance in a neurosurgical intensive care unit. J Hosp Infect. [Internet] 2006;64(1) [acesso em 08 mar 2015]. Disponível: https:// doi.org/10.1016/j.jhin.2006.02.022.

16. de Oliveira ABF, Dias OM, Mello MM, Araújo S, Dragosavac D, Nucci A, et al. Factors associated with increased mortality and prolonged length of stay in an adult intensive care unit. Rev. bras. ter. Intensive. [Internet] 2010;22(3) [acesso em 08 mar 2015]. Disponível: http://dx.doi.org/10.1590/S0103-507X2010000300006.

17. Elliott M. Readmission to intensive care: a review of the literature. Aust Crit Care. [Internet] 2006;19(3) [acesso em 08 mar 2015]. Disponível: https://www.ncbi.nlm.nih.gov/pubmed/16933636.

18. Chan KS, Tan CK, Fang CS, Tsai CL, Hou CC, Cheng KC, et al. Readmission to the intensive care unit: an indicator that reflects the potential risks of morbidity and mortality of surgical patients in the intensive care unit. Surg Today. [Internet] 2009;39(4) [acesso em 08 mar 2015]. Disponível: http://dx.doi.org/10.1007/s00595-008-38766.

19. Kramer AA, Higgins TL, Zimmerman JE. Intensive care unit readmissions in U.S. hospitals: patient characteristics, risk factors, and outcomes. Crit Care Med. [Internet] 2012;40(1) [acesso em 08 mar 2015]. Disponível: http:// dx.doi.org/10.1097/CCM.0b013e31822d751e.

20. de Araujo TG, Rieder MM, Kutchak FM, Franco Filho JW. Readmissions and deaths following ICU discharge. Rev Bras Ter Intensiva. [Internet] 2013;25(1) [acesso em 08 mar 2015]. Disponível: http://dx.doi.org/10.1590/S0103507X2013000100007.

21. Campbell AJ, Cook JA, Adey G, Cuthbertson BH. Predicting death and readmission after intensive care discharge. Br J Anaesth. [Internet] 2008;100(5) [acesso em 08 mar 2015]. Disponível: http://dx.doi.org/10.1093/bja/ aen069.

22. Goldhill DR, McMarry AF, Hadjianastassiou VG, Tekkis PP. The longer patients are in hospital before Intensive Care admission the higher their mortality. Intensive Care Med. [Internet] 2004;30(10) [acesso em 08 mar 2015]. Disponível: http://dx.doi.org/10.1007/s00134-004-2386-2. 